\title{
Electric Vehicle Modeling Utilizing DC Motor Equations
}

\author{
Clay S. Hearn, Damon A. Weeks, Richard C. Thompson, and Dongmei Chen
}

\begin{abstract}
This paper discusses modeling an electric utility vehicle powered by a separately wound DC motor. Many modeling techniques use steady state efficiency maps and torquespeed curves to describe the performance of electric motors, which can overlook transient response dynamics, current limits, and thermal limits that may affect the end vehicle performance. This paper discusses using bond-graph techniques to develop a causal model of an electric vehicle powered by a separately wound DC motor and development of the appropriate feedforward and feed-back controllers required for route following. The causal model performance is compared to a PSAT model of the same electric vehicle, which uses motor torque-speed curve and efficiency map.
\end{abstract}

Index Terms - dc motors, PSAT, bond graphs, modeling, and electric vehicle

\section{INTRODUCTION}

A 11 electric utility vehicles such as the Columbia ParCar SUV-LN, shown in Figure 1, are used in a wide variety of industrial and commercial applications where tools, equipment, and personnel need to be transported efficiently with zero emissions. A large number of these vehicles on the market are powered by brush type separately wound DC motors using $48 \mathrm{~V}$ to $72 \mathrm{~V}$ lead acid batteries for energy storage.

Under a program sponsored by the Defense Logistics Agency, DLA, the Center for Electromechanics at The University of Texas at Austin researched possible performance improvements by upgrading the stock lead acid batteries to state of the art lithium-ion batteries or integrating a hydrogen fuel cell with supplemental energy storage. Under this program, different vehicle configurations were analyzed with PSAT, which is a Matlab/Simulink based tool developed by Argonne National Laboratories. PSAT offers a large library of engine, motor, and battery component models for quickly evaluating different vehicle configurations. In addition, since PSAT is based in the Matlab/Simulink environment, the user is able to modify components and supervisory control strategy

Manuscript received February 1, 2010. This work was supported by the U.S. DOD - Defense Logistics Agency under a program administered by the Center for Transportation and the Environment.

C.S. Hearn, D.A. Weeks, and R.C. Thompson are with the Center for Electromechanics at the University of Texas at Austin (e-mail: hearn@cem.utexas.edu).

D. Chen is with the Department of Mechanical Engineering at the University of Texas at Austin (e-mail: dmchen@me.utexas.edu).

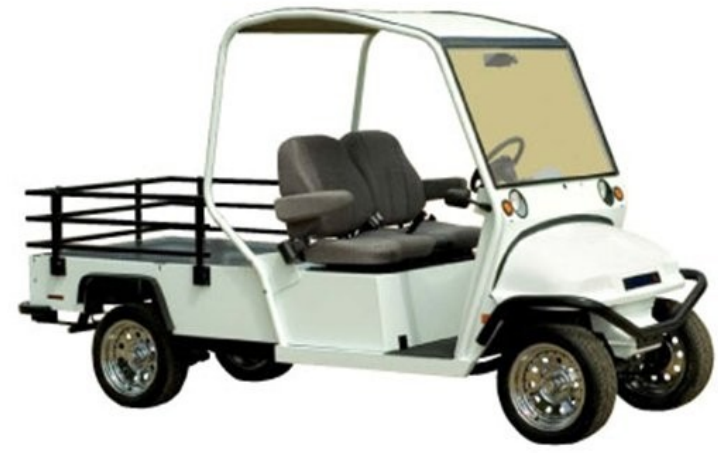

Fig. 1. Columbia ParCar SUV-LN electric utility vehicle

for particular applications. The Center for Electromechanics has successfully used PSAT in the past to predict on-route energy usage of a plug-in hydrogen fuel cell shuttle bus. Tuned PSAT model predictions of energy consumption matched data collected from on-road testing to within 5\% [1].

PSAT is considered a forward looking model since a controlled drive torque demand is used to control the vehicle following a particulate route. From the motor torque and vehicle speed, quasistatic model techniques are used to track power consumption through steady state torque speed curves and efficiency maps [2]. These quasistatic model techniques based on power flow may lose important transient dynamics which affect vehicle model performance as well as other current or thermal limit constraints, thus could limit performance.

This paper will discuss remodeling the electric utility vehicle using causal modeling relationships by replacing the torque speed curve and efficiency map used in the PSAT model with DC motor equations. Developing fully causal models of all electric vehicles has been presented in literature. Chen et al. discussed methods of causal modeling of an electric vehicle driven by a permanent magnet synchronous machine, where the motor controller switching functions and modulation vectors are actively controlled to drive the vehicle along a route [3]. The modeling techniques used Energetic Macroscopic Representation (EMR) to define the system equations and used inversion based control rules to determine the switching requirements for the vehicle model [4]. The methods presented in this paper will rely on bond-graph representation of the system and development of appropriate feed-forward and feedback controllers to control the DC motor. The base vehicle for this analysis is the Columbia ParCar SUV-LN which utilizes a $12.9 \mathrm{~kW}$, separately excited, 
DC motor. Performance of the developed causal model will be compared to the original PSAT model to assess any differences in possible energy consumption predictions.

\section{Model DeVelopment}

\section{A. Vehicle Description}

The Columbia ParCar SUV-LN is the base vehicle for this analysis and parameters for the DC motor are derived from vendor test data. General specifications for the vehicle are shown in Table I. This utility vehicle is powered by a 12.9 $\mathrm{kW}$ peak separately wound DC motor with a $400 \mathrm{amp}$ current limit controller. The stated curb weight of the vehicle is 794 $\mathrm{kg}$, but $900 \mathrm{~kg}$ is used as a loaded vehicle weight in the following analyses.

Table I. Vehicle specifications for Columbia ParCar SUV-LN

\begin{tabular}{|l|c|}
\hline Peak Motor Power & $12.9 \mathrm{~kW}$ \\
\hline Gear Ratio & 10.35 \\
\hline Tires & $175 / 50 \mathrm{R} 13$ \\
\hline Battery & $48 \mathrm{~V}-215 \mathrm{AH}$ \\
& Lead-Acid \\
\hline Curb Weight & $794 \mathrm{~kg}$ \\
\hline Cargo Capacity & $447 \mathrm{~kg}$ \\
\hline
\end{tabular}

\section{B. Model Equations}

The DC motor for the SUV-LN is a separately excited machine, which means that the voltages to the field and armature windings are independently controlled via a voltage limiting device. Figure 2 shows a simplified schematic model used to describe the connections of the field and armature windings to the output voltage of the vehicle batteries for model development. A simple equivalent circuit battery model is used, which contains an SOC (State of Charge) dependent open circuit voltage and internal resistance. More complex batteries models could be used to characterize transient dynamics. The electromagnetic torque generated by the motor is transferred to the drive shaft of the vehicle.

The electrical inputs to the field and armature windings are the field voltage and armature voltage, $\mathrm{V}_{\mathrm{f}}$ and $\mathrm{V}_{\mathrm{a}}$, which are supplied from the battery and regulated by a DC converter within the vehicle motor controller. The basic DC converter consists of a diode and a high speed switch (mosfet, thyristor, IGBT, or similar device) which is rapidly turned on and off over a specified duty cycle [5]. For a step-down converter, the duty cycle, $\mathrm{D}$, can be characterized as a value between 0 to 1 that decreases the input voltage by the equivalent percent.

For an idealized DC converter with no losses, and operating over duty cycle $\mathrm{D}$, the device will act as a transforming element in bond graph theory. For example, equations (1) and (2) can be used to characterize the input and output voltages and currents delivered by the battery and supplied to the motor armature:

$$
\begin{aligned}
& V_{a}=D V_{b a t t} \\
& I_{b a t t}=D I_{a}
\end{aligned}
$$

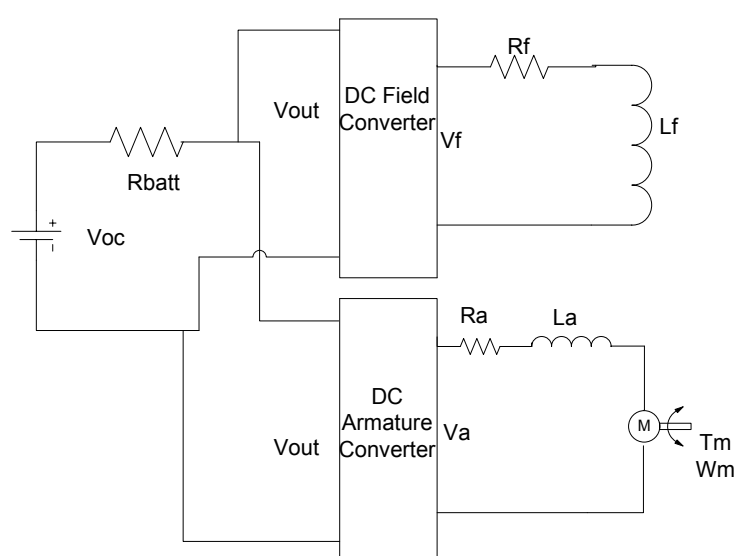

Fig. 2. Simplified electrical schematic for vehicle powered by separately wound DC motor

Similar equations would be used for the field current and voltage. The total current supplied by the battery would be a sum of the currents delivered to the armature and field windings. For the model presented in this paper, the DC motor converters are assumed as ideal with no losses. Current flow is also assumed to be bi-directional to allow for regenerative braking. If more information was known about the specific circuit parameters of the field and armature converters, this information could be incorporated into the final equations. For now, this paper characterizes the converters as ideal transforming elements where the switching duty cycles will be the vehicle control parameters.

Typically, the control of a DC motor can be separated into two different operating regimes as shown in Figure 3. Up to base speed, a constant field current is applied to yield constant torque, whereby the armature voltage is controlled for speed control. Above base speed, the field current is reduced, also known as field weakening, to achieve higher motor speeds [6].

A labeled bond graph representation with causality strokes of the electrical vehicle is shown in Figure 4. Bond graphs show the power flow transfer through different energy

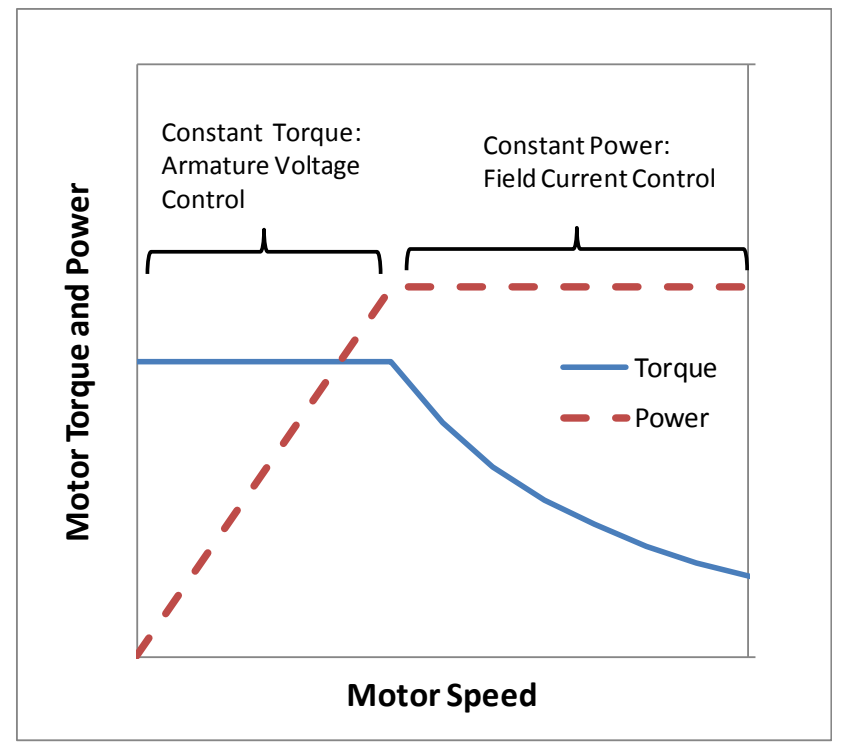

Fig. 3. Torque and power curves for typical DC motor control 


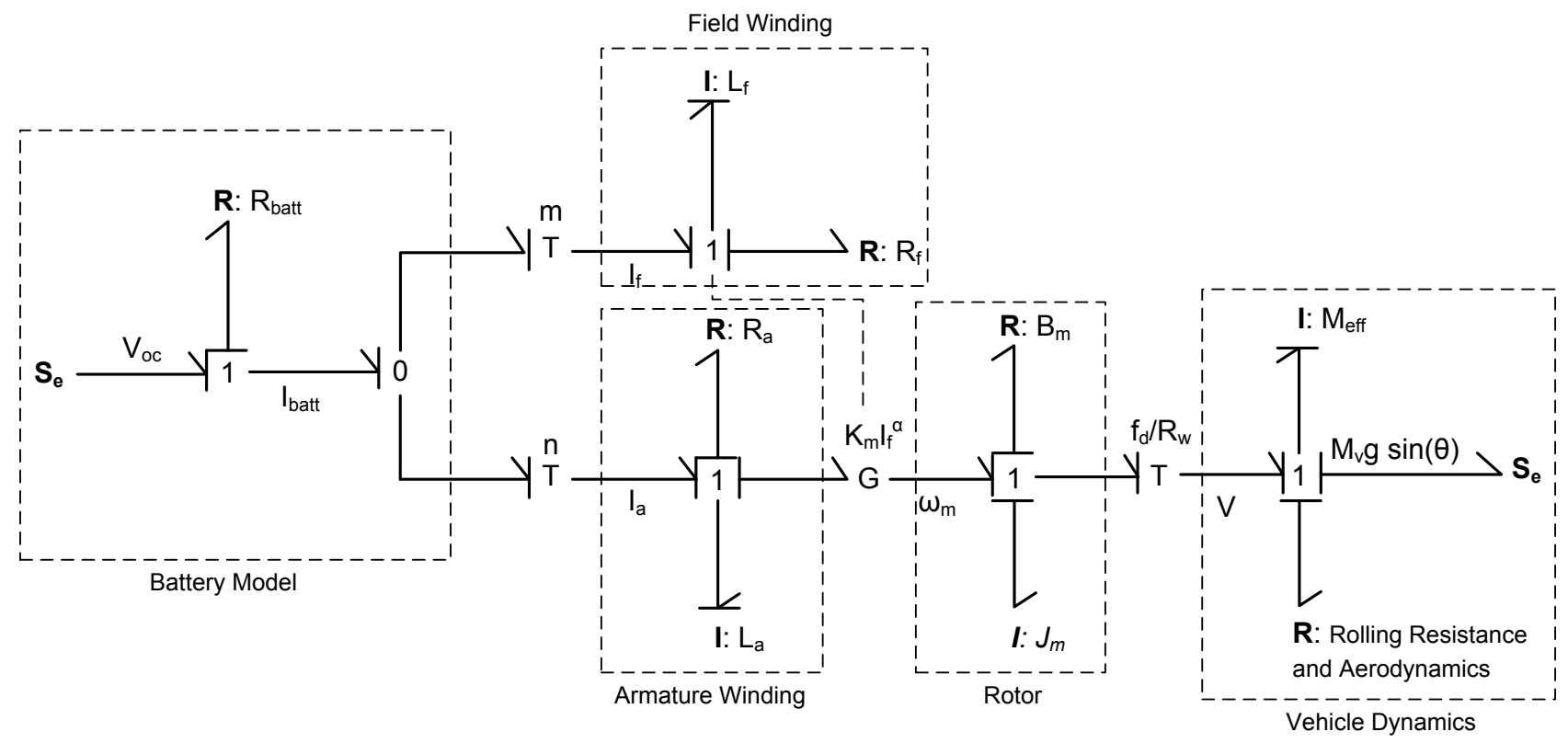

Fig. 4. Bond graph representation of electric utility vehicle powered by a separately wound DC motor.

domains and the causality of the system [7]. From the bond graph, the output battery voltage is transferred to the field and armature windings of the DC motor through respective transfer ratios, $m$ and $n$. The transfer ratios $m$ and $n$ represent the switching duty cycle demand of an ideal DC converter with ratio values operating between 0 and 1 (i.e. output battery voltage can only be reduced to motor windings). Control of these ratio values will be used to drive the vehicle along a specified route profile. The electromagnetic torque developed by the armature current is transferred through the drive gear and wheels as a tractive effort overcoming longitudinal inertia, aerodynamic, rolling resistance and grade losses.

Radcliffe showed the DC motor constant can be modeled as a nonlinear function of the field current which characterizes saturation effects of the motor [8]. As field current is increased, the amount of gain in flux and relevant motor constant starts to decrease, which can be described by a power-law fitting of the data. The DC motor constant, $\mathrm{K}$, will be characterized as a function of field current per equation (3) in this paper, where $\mathrm{K}_{\mathrm{m}}$ and $\alpha$ are constants determined from curve fitting vendor data.

$$
K=K_{m} I_{f}^{\alpha}
$$

From the bond graph shown in Figure 4, the following five equations can be derived to describe the electric vehicle performance through the following four states, battery SOC, field current, $I_{f}$, armature current, $I_{a}$, and vehicle velocity, $V$. The duty cycles, $\mathrm{m}$ and $\mathrm{n}$, are the active controls that regulate battery output voltage to the field and armature winding circuits. $\quad M_{e f f}$ is the effective vehicle inertia, including the vehicle mass, $M_{v}$, and the inertial effects of the rotating components (rotor and wheels).

$$
V_{\text {out }}=V_{\text {oc }}(S O C)-I_{\text {batt }} R_{\text {batt }}(S O C)
$$

$$
\begin{gathered}
S O C=\frac{\int I_{\text {batt }} d t}{C_{b}} \\
\dot{I}_{f}=\frac{m V_{\text {out }}}{L_{f}}-\frac{R_{f}}{L_{f}} I_{f} \\
\dot{I}_{a}=\frac{n V_{\text {out }}}{L_{a}}-\frac{R_{a}}{L_{a}} I_{a}-\frac{K_{m} I_{f}^{\alpha}}{L_{a}} \frac{f_{d}}{R_{w}} V \\
\dot{V}=\frac{f_{d}}{R_{w}} \frac{K_{m} I_{f}^{\alpha}}{M_{e f f}} I_{a}-\frac{B_{m}}{M_{e f f}}\left(\frac{f_{d}}{R_{w}}\right)^{2} V-\frac{1 / 2 \rho C_{d} A_{f}}{M_{e f f}} V^{2} \\
-\frac{M_{v} g}{M_{e f f}}\left(\sin \theta+f_{r} \cos \theta\right)
\end{gathered}
$$

\section{Controller Design}

From equations (6) and (7), the primary control parameters are the DC regulating variables $\mathrm{m}$ and $\mathrm{n}$, which control the applied field and armature voltages, respectively. In order to control the vehicle along a specified route profile, controllers must be developed along with estimators to determine reference torque and current requirements. An initial driver model is developed to estimate torque requirements from a given route profile, and the motor controller model transfers the torque requirements into field and armature current demands.

The developed electromagnetic torque, which is defined by the nonlinear term $K_{m} I_{f}^{\alpha} I_{a}$ in equation (8), drives the vehicle along the path. In order to determine the required torque for matching a specific velocity profile a driver model is developed to estimate the required torque. The torque estimator design utilizes a feed-forward component, based on the velocity demand, and a feedback PI controller to correct differences between the vehicle velocity and reference profile velocity. 
The design of the feed-forward component is based on the set of linearized equations for longitudinal vehicle motion from equation (8). Linearization about a specified $\mathrm{V}_{0}$ yields the equation (9).

$$
\begin{gathered}
M_{e f f} \Delta \dot{V}=\left(\frac{f_{d}}{R_{w}}\right) \Delta \tau_{e m}-\left(B_{m}\left(\frac{f_{d}}{R_{w}}\right)^{2}+\rho C_{d} A_{f} V_{0}\right) \Delta V \\
-M_{v} g\left(f_{r} \sin \theta-\cos \theta\right) \Delta \theta
\end{gathered}
$$

The steady state electromagnetic torque, $\tau_{\text {emo }}$ is defined by setting $\Delta \dot{V}$ equal to zero.

$$
\begin{gathered}
\tau_{e m 0}=B_{m}\left(\frac{f_{d}}{R_{w}}\right) V_{0}+ \\
\left(\frac{R_{w}}{f_{d}}\right)\left(1 / 2 \rho C_{d} A_{f} V_{0}^{2}+M_{v} g\left(\sin \theta_{0}+f_{r} \cos \theta_{0}\right)\right)
\end{gathered}
$$

From equation (9), a transfer function between the input EM motor torque, $\tau_{e m}$, and vehicle velocity can be derived as shown in equation (11).

$$
\frac{\Delta V(s)}{\Delta \tau_{e m}(s)}=\frac{\left(\frac{f_{d}}{R_{w}}\right)}{M_{e f f} s+\left(B_{m}\left(\frac{f_{d}}{R_{w}}\right)^{2}+\rho C_{d} A_{f} V_{0}\right)}
$$

Equation (11) shows a linearized transfer function for a given input torque to output a velocity, but for the feedforward controller, the controller needs to return a torque demand for a given change in reference speed. Equation (11) is hence inverted to yield equation (12).

$$
\frac{\Delta \tau_{e m}(s)}{\Delta V(s)}=\left(\frac{R_{W}}{f_{d}}\right) M_{e f f} S+\left(\frac{R_{W}}{f_{d}}\right)\left(B_{m}\left(\frac{f_{d}}{R_{w}}\right)^{2}+\rho C_{d} A_{f} V_{0}\right)
$$

Equation (12) is a transfer function with an output torque dependent on an input velocity, coming from the simulated route profile. This transfer function has one zero and requires a unit gain low pass filter for stability. The vehicle speed range is between 0 to $11 \mathrm{~m} / \mathrm{s}$, which yields the corresponding zero locations shown in Table II.

Since there is little variance in the zero location, a unity filter is selected with a pole located 100 times to the left of the zero at $\mathrm{V}_{0}=10 \mathrm{~m} / \mathrm{s}$. The feed-forward transient speed

Table II. Locations for linearized feed-forward controller

\begin{tabular}{|c|c|}
\hline Velocity $[\mathrm{m} / \mathrm{s}]$ & Zero Location \\
\hline 2 & -0.042 \\
\hline 6 & -0.051 \\
\hline 10 & -0.060 \\
\hline
\end{tabular}

component of the torque estimator now has the following form:

$$
\frac{\Delta \tau_{e m}(s)}{\Delta V(s)}=\frac{6\left[\left(\frac{R_{w}}{f_{d}}\right) M_{e f f} s+\left(\frac{R_{w}}{f_{d}}\right)\left(B_{m}\left(\frac{f_{d}}{R_{w}}\right)^{2}+\rho C_{d} A_{f} V_{0}\right)\right]}{s+6}
$$

The stationary component of the feed-forward torque estimator, equation (14) calculates the steady speed torque requirement minus the steady state component of the dynamic torque estimator from equation (13). The final driver model is a summation of equations (13) and (14) for a given time dependent route profile plus a feedback PI controller that compares desired speed to actual speed for additional torque corrections.

$$
\begin{gathered}
\tau_{\text {em } 0}=B_{m}\left(\frac{f_{d}}{R_{w}}\right) V \\
+\left(\frac{R_{w}}{f_{d}}\right)\left(1 / 2 \rho C_{d} A_{f} V^{2}+M_{v} g\left(\sin \theta_{0}+f_{r} \cos \theta_{0}\right)\right) \\
-\left(\frac{R_{w}}{f_{d}}\right)\left(B_{m}\left(\frac{f_{d}}{R_{w}}\right)^{2}+\rho C_{d} A_{f} V_{0}\right)
\end{gathered}
$$

From the desired EM torque demand, $\tau_{e m}$, the armature and field currents are calculated and controlled via separate PI controllers. For operations below motor base speed of 2000 rpm, the field current is arbitrarily set to a constant $10 \mathrm{amps}$, and the required armature current is calculated from the torque demand of the driver model.

$$
I_{a}=\frac{\tau_{e m}}{K_{m} I_{f}^{\alpha}}
$$

A maximum armature current limit is set to 400 amps per vehicle motor controller specifications. Extra limits on current draw due to thermal considerations are not considered at this time, but could easily be included into the model via a thermal lumped capacitance motor model. Above base speed, the field current is weakened, which requires calculating appropriate current strength based on the states of the system. Equations (7) and (8) are rewritten to determine steady state operating points of field and armature current based on fixed armature voltage command $\mathrm{N}$ (equation (8) is also rewritten with respect to motor speed, $\omega_{m}$, rather than vehicle speed, $\left.\mathrm{V}\right)$.

$$
\begin{gathered}
N V_{\text {out }}-R_{a} I_{a}-K_{m} I_{f}^{\alpha} \omega_{m}=0 \\
K_{m} I_{f}^{\alpha} I_{a}-B_{m} \omega_{m}-1 / 2 \rho C_{d} A_{f}\left(\frac{R_{w}}{f_{d}}\right)^{3} \omega_{m}^{2} \\
-M_{v} g\left(\sin \theta+f_{r} \cos \theta\right)\left(\frac{R_{w}}{f_{d}}\right)=0
\end{gathered}
$$

From the above equations, the armature current in equation (15) can be rewritten as:

$$
I_{a}=\frac{N V_{o u t}}{R_{a}}-\frac{K_{m} I_{f}^{\alpha} \omega_{m}}{R_{a}}
$$

Substituting equation (17) into (16) yields the following polynomial shown in equation (18). 


$$
\begin{aligned}
\left(\frac{-K_{m}^{2} \omega_{m}}{R_{a}}\right) I_{f}^{2 \alpha}+ & \left(\frac{K_{m} N V_{\text {out }}}{R_{a}}\right) I_{f}^{\alpha} \\
& -\left(B_{m} \omega_{m}+1 / 2 \rho C_{d} A_{f}\left(\frac{R_{w}}{f_{d}}\right)^{3} \omega_{m}^{2}\right. \\
& \left.+M_{v} g\left(\sin \theta+f_{r} \cos \theta\right)\left(\frac{R_{w}}{f_{d}}\right)\right)=0
\end{aligned}
$$

This polynomial equation can be solved for $I_{f}^{\alpha}$ dependent upon a specified motor speed, battery output voltage, and armature switching command. Once the reference field and armature currents have been calculated, standard feedback PI controllers are used to regulate the model currents. These controllers are tuned in the Simulink model.

\section{Simulation AND Results}

An overview of the entire vehicle model implemented in Simulink is shown in Figure 5. From a given speed and grade profile, the driver model estimates a required motor torque for the motor controller and passes through the relevant motor speed to control function between constant field and field weakening operation. The controller field and armature voltage regulation commands are then sent to the vehicle model which analyzes the primary model equations, (4) through (8).

The model was analyzed against an applicable route profile developed for an electric utility vehicle (EUV) and compared to vehicle performance from the original PSAT study. The EUV route profile has a maximum speed of approximately 8 $\mathrm{m} / \mathrm{s}(18 \mathrm{mph})$ with ten stops and starts along the 1.4 mile length. The distances between stops varies between 150 to $300 \mathrm{~m}$. Vehicle route following performance and current demands are shown in Figures 6 and 7 for a small section along the route. One start and stop interval is shown in these figures for clarity.

Overall, the model matches the demand route well with little significant deviation. Figure 7 shows vehicle speed and relevant battery current for one stop and start interval on the route. The positive battery currents represent battery discharge current, which drains the battery SOC, while negative currents represent battery charging currents from regenerative braking of the vehicle resulting in negative armature currents from the DC motor. For this model, all braking is assumed to come from the motor, and mechanical brakes are not included. Figure 7 shows the relevant armature and field currents, along with the duty cycle commands over the same start and stop interval shown in Figure 6.

Figure 8 compares the model performance using the causal DC motor equations and control strategy developed in this paper to the original PSAT predictions on energy consumption. Figure 8 shows after one lap on the given route there is about a $0.75 \%$ difference in SOC estimation, which can become a more significant difference along longer routes and runtimes. The original PSAT model used a table-based efficiency map scaled to appropriate torque and speed limits, while the DC motor model utilized available vendor test data and given resistance parameters to develop a dynamic model. When tracking energy consumption is the primary modeling goal, this comparison shows the magnitude of possible modeling errors which could exist between the two methods. For accurate model development, the model needs to be compared and tuned to full vehicle performance test data.

The actual motor controller on the ParCar-SUV-LN will limit the motor current based on the measured motor temperature to prevent overheating. This effect could be easily incorporated into this model to replicate actual load dependent performance limits the vehicle may encounter

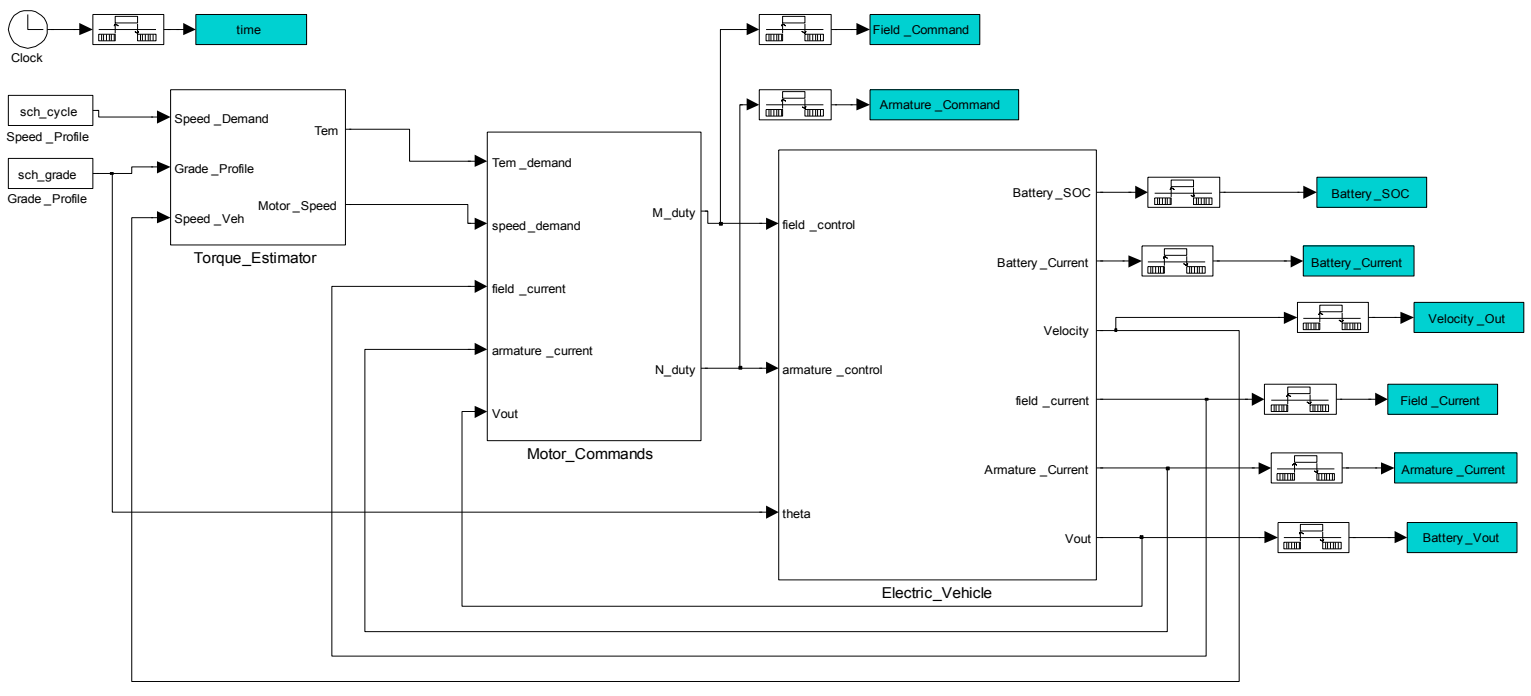

Fig. 5. Complete Simulink model of electric utility vehicle and controls 

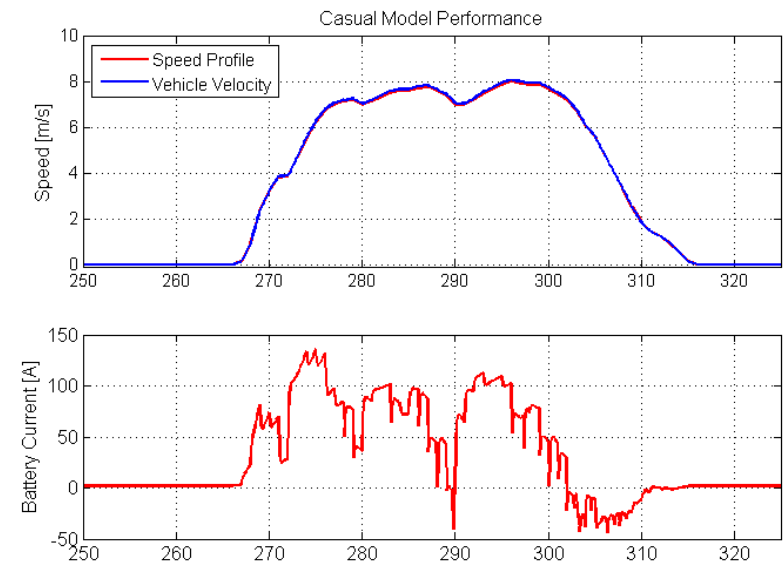

Fig. 6. Vehicle route following performance between start to stop interval
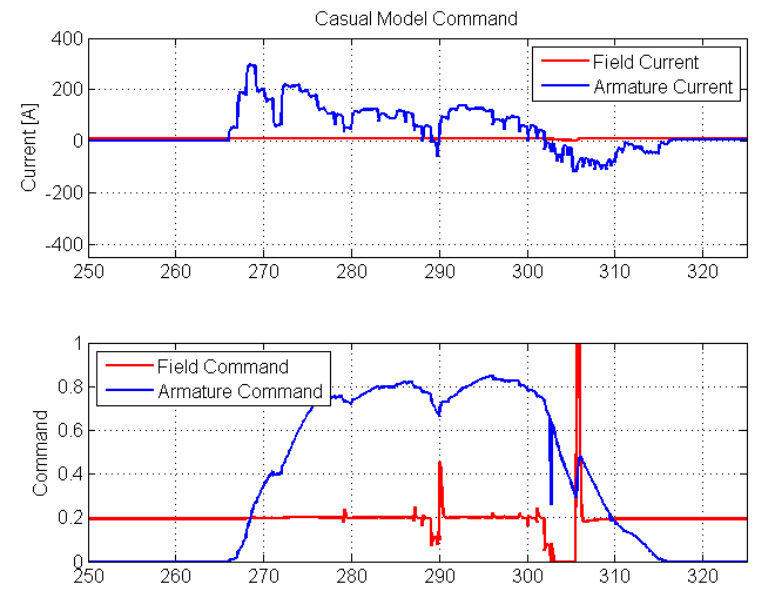

Fig. 7. Field and armature currents and commands over start to stop interval.

under high power conditions such as climbing a steep grade.

More advanced control schemes could also be incorporated into this model. The primary DC motor dynamic equations, equations (6) to (8) are nonlinear, especially during field weakening operation. Liu discussed using advanced nonlinear control techniques to control field weakening DC motors above base speeds. These techniques include feedback linearization and development of a MIMO field weakening controller which could be applied to the current vehicle model for improved control response $[9,10]$. Other techniques that focus on optimization such as dynamic programming can also be applied to reduce energy usage and develop more efficient control algorithms.

\section{CONCLUSION}

A fully causal model of an electric vehicle driven by a separately excited DC motor was presented in this paper along with a method for developing a feed-forward driver controller and appropriate motor reference currents. The simulation result shows the model has good route following characteristics. It also indicates potential improvement on the SOC estimation and the energy consumption prediction,

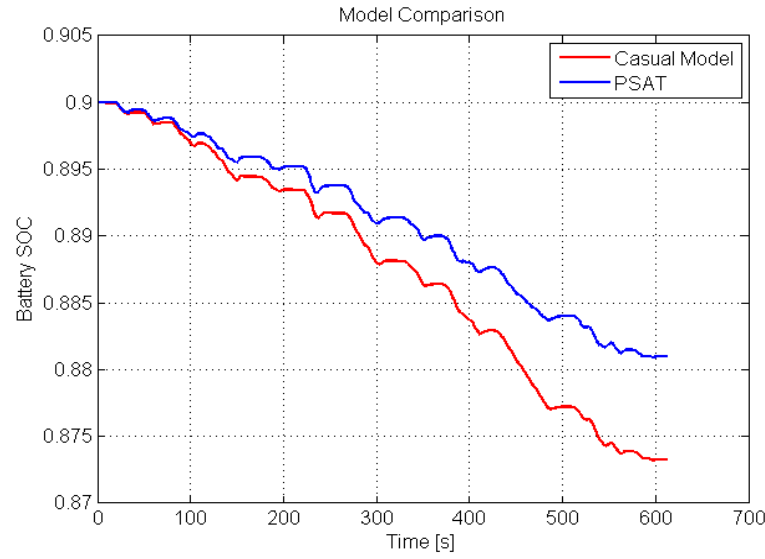

Fig. 8. Performance of causal model utilizing DC motor equations to PSAT model which utilizes steady state efficiency look-up maps.

provided appropriate motor parameters and control strategies are utilized. In order to determine the significance of this prediction benefit, the model should be compared to experimental data. To date, no experimental data has been recorded, but vehicle testing will start under a US-DOD DLA sponsored program during the summer of 2010. For further enhancing the system performance, the development of the motor controller with this method allows for implementation of more advanced control techniques or imposition of additional limits.

\section{ACKNOWLEDGMENT}

The authors would like to thank the Center for Transportation and the Environment for their support under the US DOD program which provided the technical foundation for this paper's research, and Columbia ParCar for their technical support and assistance.

\section{REFERENCES}

[1] C.S. Hearn, M.C. Lewis, R.C. Thompson, and R.G. Longoria. Modeling and Evaluation of a Plug-In Hybrid Fuel Cell Shuttle Bus. Vehicle Power and Propulsion Conference, IEEE, VPPC 2008, Dearborn Mi.

[2] L. Guzzella and A. Sciarretta. Vehicle Propulsion Systems. 2nd Ed., Springer, New York, 2007.

[3] K. Chen, P. Delarue, A. Bouscayrol, and R. Trigui. Influence of Control Design on Energetic Performances of an Electric Vehicle. IEEE 0-78039761-4/07

[4] P.J. Barre, A. Bouscayrol, et al. Inversion Based Control of Electromechanical Systems Using Causal Graphical Descriptions. IEEE $1-4244-0136-4 / 06$

[5] Mohan, Undeland, and Robbins. Power Electronics: Converters, Applications, and Design. $3^{\text {rd }}$ Ed. Media Update CD, John Wiley and Sons Inc. 2003

[6] Fitzgerald, A.E., Kingsley, Charles Jr., and Umans, Stephen D. Electric Machinery. $6^{\text {th }}$ Ed. McGraw Hill. Boston, 2003

[7] Karnopp, Dean C., Margolis, Donald, L., Rosenberg, Ronald C. System Dynamics: Modeling and Simulation of Mechatronic Systems. John Wiley \& Sons, Inc. New York, 2000.

[8] C. Radcliffe. An Analytical Mechatronic Model for Series DC Motors Using Manufacturer Test Data. Proceedings of the ASME 2009 Dynamic Systems and Control Conference, Oct 12-14, 2009.

[9] Luo Fang Lin, Liu Zou Zong, and D. Tien. Nonlinear Field Weakening Controller of a Separately Excited DC Motor. IEEE 0-7803-4495-2/98.

[10] Zuo Z. Liu, Fang, L. Luo, and Muhammad H. Rashid. High Performance Nonlinear MIMO Field Weakening Controller of a Separately Excited DC Motor. Electric Power Systems Research, Vol. 55 (2000) pg 157-164. 